\title{
Bloqueio de nervo safeno e nervo ciático para síndrome dolorosa complexa regional tipo II em membro inferior
}

\section{Saphenous and ciatic nerves blockade in order to treat type II Complex Regional Pain Syndrome in lower limb}

\author{
Camila de Melo Matos Lins Beccoํ․ Josenília Maria Alves Gomes². \\ 1 Instituto Dr José Frota, Fortaleza, Ceará, Brasil. 2 Hospital Universitário Walter Cantídio (HUWC), Universidade Federal do \\ Ceará (UFC), Fortaleza, Ceará, Brasil.
}

\section{RESUMO}

A Síndrome Dolorosa Complexa Regional (SDCR) é uma desordem dolorosa que leva a sério comprometimento na qualidade de vida dos pacientes acometidos e que, muitas vezes, necessita de abordagens mais invasivas para obter algum conforto. A técnica clássica para abordagem da SDCR em membros inferiores, ou seja, o bloqueio do gânglio simpático lombar, apesar de ser efetiva, traz algumas complicações que podem ser atenuadas ou mesmo evitadas com técnicas mais modernas e mais baratas como o bloqueio de nervos periféricos guiados por ultrassonografia. O objetivo do presente artigo é relatar o caso de um paciente com SDCR tipo II em membro inferior refratário à terapia medicamentosa que obteve sucesso terapêutico através do bloqueio dos nervos safeno e ciático guiados por ultrassonografia.

Palavras-chave: Síndrome Dolorosa Complexa Regional. Síndrome Dolorosa Complexa Regional tipo II. Ultrassonografia intervencionista. Bloqueio de nervos. Dor neuropática.

\begin{abstract}
The Complex Regional Pain Syndrome (CRPS) is a painful condition that leads to severe decrease of patient's quality of life and, frequently, demands more invasive procedures to achieve some relieve. The traditional management to treat CRPS in lower limbs, it means, the lumbar sympathetic block, although effective, brings some complications that can be healed or even avoided with more recent and cheaper approaches as ultrasound-guided peripheral nerve block. The purpose of this article is to report the case of a patient with lower limbs CRPS type 2 refractory to medication that obtained therapeutic success due to ultrasound-guided saphenous and sciatic nerve blocks.
\end{abstract}

Keywords: Complex Regional Pain Syndromes. Complex Regional Pain Syndrome Type II. Ultrasound, interventional. Nerve blockade. Neuropathic pain.

Autor correspondente: Camila de Melo Matos Lins Becco, Marcos Macedo, 1460, Aldeota, Fortaleza, Ceará. CEP: 60150-190. Telefone: +55 85 98200-3006. E-mail: camilalinsce@hotmail.com

Conflito de interesses: Não há qualquer conflito de interesses por parte de qualquer um dos autores.

Recebido em: 19 Abr 2018; Revisado em: 01 Out 2018; Aceito em: 03 Out 2018. 


\section{INTRODUÇÃO}

A Síndrome Dolorosa Complexa Regional (SDCR) é uma síndrome dolorosa neuropática, caracterizada por dor intensa em uma extremidade, desproporcional ao evento desencadeante. ${ }^{1} \mathrm{O}$ diagnóstico é essencialmente clínico. $\mathrm{O}$ paciente deve apresentar pelo menos um sinal/sintoma em três das seguintes categorias: alterações sensoriais (hiperestesia/ alodínea), vasomotoras (da temperatura ou da sudorese do membro afetado comparado ao não acometido), sudomotoras/ edema e motoras/tróficas (das unhas, cabelos ou pele). Nenhum outro diagnóstico justifica os sinais e sintomas do paciente. ${ }^{2}$ A SDCR é classificada em dois tipos: I e II, diferem entre si basicamente pela presença de lesão nervosa real, presente no subtipo II e ausente no $\mathrm{I}^{3}{ }^{3}$ Os eventos mais relacionados ao desenvolvimento dessa síndrome são fraturas, entorses e imobilizações, mas até 7\% dos casos ocorrem sem nenhum trauma conhecido. ${ }^{4,5}$ As fraturas lideram como principal fator desencadeante, ${ }^{6}$ e a razão entre homens e mulheres varia de 1:2 e 1:4. A evolução dessa síndrome tende a ser pior em pacientes com lesões nas extremidades superiores comparado àqueles com lesões em membros inferiores (MMII), lesões outras que não fraturas, na SDCR "fria" (crônica) comparada à SDCR "quente" (aguda) e naqueles que apresentam lesão de nervos motores em relação àqueles com lesão de nervos sensitivos. ${ }^{6} \mathrm{~A}$ idade média dos pacientes é em torno de 41,6 anos. ${ }^{1}$

Essa síndrome causa grande prejuízo na qualidade de vida dos indivíduos acometidos. O tratamento é dificultado pela escassez de conhecimentos sobre sua etiologia, limitando o desenvolvimento de terapias mais direcionadas para sua abordagem. ${ }^{1}$ A maioria das medicações de primeira-linha no tratamento da SDCR tem sido investigadas na abordagem de outras desordens neuropáticas e então aplicadas para SDCR com resposta terapêutica variável. As modalidades terapêuticas predominantes são fisioterapia, agentes farmacológicos e procedimentos intervencionistas. ${ }^{1}$

O presente trabalho visa relatar o caso de um paciente com SDCR tipo II em membro inferior refratário à terapia medicamentosa que obteve sucesso terapêutico através de uma abordagem intervencionista da dor por meio de bloqueio dos nervos safeno e ciático guiados por ultrassonografia.

\section{RELATO DE CASO}

Paciente do sexo masculino, 40 anos, encaminhado para clínica da dor em maio de 2012 com queixa de dor intensa (Escala Visual Numérica (EVN) de 8), alodínea importante, e parestesia e paresia no pé esquerdo 6 meses após cirurgia para tratamento de fratura exposta no tornozelo esquerdo por queda da própria altura. Foram realizadas três cirurgias entre outubro e dezembro do mesmo ano para tratamento da fratura. A dor era descrita como em "pontadas", de caráter constante, com significativo comprometimento na realização das atividades de vida diária. Fazia uso de codeína com paracetamol e carbamazepina, mas a resposta analgésica era insatisfatória, além de apresentar muitos efeitos colaterais. A carbamazepina foi substituída pela gabapentina, cuja dose foi aumentada gradualmente até atingir $1200 \mathrm{mg} / \mathrm{dia}$. Nas consultas subsequentes, em virtude da persistência do quadro álgico, foi associada amitriptilina até atingir dose de $50 \mathrm{mg} /$ dia e a associação codeína-paracetamol foi substituído por codeína, medicação que chegou a usar na dose de $240 \mathrm{mg} /$ dia. Realizou eletroneuromiografia, cujo laudo mostrou "mononeuropatia sensitivo-motora, axonal, de nervo tibial posterior direito a nível de tornozelo".

Diante da ausência de melhora do quadro álgico durante os 2 anos de tratamento clínico, em novembro de 2014, optou-se por indicar bloqueio analgésico do nervo ciático e do nervo safeno guiados por ultrassom, baseados nas áreas com maior intensidade da dor e da alodínea referidas pelo paciente. Esses sintomas correspondiam aos dermátomos inervados por esses dois nervos. Os bloqueios eram semanais durante o $1^{\circ}$ mês, quinzenais durante os dois meses seguintes e mensais daí em diante. A solução analgésica consistia em $8 \mathrm{~mL}$ de levobupivacaina a $0,5 \%, 50 \mathrm{mcg}$ de fentanil, $150 \mathrm{mcg}$ de clonidina e $3 \mathrm{ml}$ de água destilada, totalizando $15 \mathrm{ml}$ de solução distribuídos meio a meio para bloqueio dos nervos safenos e ciático. Paciente relatou melhora significativa da dor já no primeiro bloqueio, com EVN de 9 antes do bloqueio para EVN de 0 imediatamente após bloqueio. No quarto dia, sentiu desconforto, atribuindo EVN de 3 para queixa. Nos bloqueios subsequentes, manteve boa resposta analgésica, mesmo após período mais extenso entre bloqueios (repetição mensal). Diante da melhora, foi iniciado desmame da codeína, reduzida a dose da gabapentina para $2 x /$ dia e da amitriptilina para 25 $\mathrm{mg}$ /dia. Manteve essa prescrição até o quinto mês após início dos bloqueios, momento em que foi relatado esse caso (abril de 2015). O paciente teve bom controle álgico durante os cinco meses de tratamento, com EVN entre 1 e 2, e melhora importante na qualidade de vida.

\section{DISCUSSÃO}

Múltiplas modalidades terapêuticas têm sido estudadas por suas habilidades em interromper a via simpática, pois acredita-se que haja participação desse sistema na perpetuação da dor neuropática da SDCR. ${ }^{7}$

Os bloqueios mais utilizados para abordagem da SDCR em membros superiores e inferiores são, respectivamente, bloqueio do gânglio estrelado e bloqueio do gânglio simpático lombar. ${ }^{8}$

O bloqueio simpático lombar é indicado para diagnóstico, prognóstico e tratamento de condições associadas a disfunções simpáticas como a SDCR tipos I e II, herpes zóster, dor do membro fantasma e doença vascular periférica inoperável de membros inferiores.

Apesar de efetivo no alívio da dor em muitos pacientes, alguns fatores tornam a aplicabilidade do bloqueio do plexo lombar pouco atraente. Para realizá-lo, o paciente deve ser posicionado em decúbito ventral com um coxim abaixo do abdômen pra fletir a coluna lombar. É realizado sob fluoroscopia impondo à 
equipe que realiza esse procedimento uma constante exposição à radiação e seus efeitos deletérios a longo prazo. ${ }^{9}$ Em virtude da proximidade das raízes nervosas e da medula espinhal, esse procedimento deve ser realizado por profissionais habilitados na anatomia da região o que requer uma curva de aprendizado mais demorada para realização com segurança desse procedimento. Outras desvantagens são: risco potencial de lesão de vísceras abdominais (proximidade da cavidade peritoneal); injeção epidural, subdural ou subaracnoide da solução; trauma de disco intervertebral, medula espinhal e raízes nervosas e infecção (por exemplo, discite). ${ }^{9}$

Uma alternativa ao bloqueio simpático lombar, consiste no bloqueio de nervos periféricos guiados por ultrassonografia. Além de dispensar a utilização de fluoroscopia, não está associado à ocorrência dos eventos adversos relacionados ao bloqueio do plexo lombar, a curva de aprendizado é menor e o conforto para o paciente superior, visto que pode ser feito em nível ambulatorial e não requer decúbito ventral para realização.

As técnicas de bloqueios nervosos guiados por ultrassom baseiam-se na visualização direta das estruturas nervosas, da agulha de bloqueio e das estruturas anatômicas adjacentes. Desta maneira, é possível depositar a solução de anestésico local e adjuvantes em torno dos nervos acometidos e acompanhar a sua dispersão em tempo real, obtendo-se, assim, um bloqueio mais eficaz e seguro. Para SDCR em MMII podemos realizar os bloqueios dos nervos ciáticos ou suas subdivisões, tibial e fibular comum, associado ao bloqueio do nervo femoral ou do seu ramo, o nervo safeno, pois essas estruturas nervosas são responsáveis pela inervação sensitiva e motora dos MMII. O bloqueio do nervo ciático em associação ao nervo safeno guiados por ultrassonografia tem sido utilizado para prover anestesia e analgesia pós-operatória para cirurgias em MMII. Esses bloqueios também têm sido utilizados como ferramentas diagnósticas para avaliar se, diante da realização de um bloqueio neural diferencial, baseado nos referenciais anatômicos, a dor é decorrente de determinado nervo. ${ }^{8}$

\section{REFERÊNCIAS}

1. Benzon H, Rathmell J, Wu CL, Turk DC, Argoff CE, Hurley RW. Pratical Management of Pain. 5. ed. Philadelphia: Elsevier; 2014. $1115 \mathrm{p}$.

2. Harden RN. Objectification of the diagnostic criteria for CRPS. Pain Med. 2010;11(8):1212-5.

3. Toth C, Moulin DE. Neuropathic pain: causes, management and understanding. New York: Cambrigde University Press; 2013. 368p.

4. Schwartzman RJ, Erwin KL, Alexander GM. The natural history of complex regional pain syndrome. Clin J Pain. 2009;25(4):273-80.

5. de Rooij AM, Perez RS, Huygen FJ, van Eijs F, van Kleef M, Bauer $\mathrm{MC}$, et al. Spontaneous onset of complex regional pain syndrome. Eur J Pain. 2010;14(5):510-3.

6. Demir SE, Ozaras N, Karamehmetoglu SS, Karacan I, Aytekin
Poucos estudos relacionam utilização dos bloqueios de nervos periféricos em MMII e terapia intervencionista da dor. Um estudo mostrou que o bloqueio do nervo ciático contínuo tem sido usado para analgesia e simpatólise em pacientes com dor induzida por isquemia. ${ }^{10}$ Um outro, de 2009, mostrou que o bloqueio único ou contínuo do nervo femoral pode ser usado com o bloqueio do ciático para dor crônica e tratamento de úlcera de pressão. ${ }^{11,12}$

No caso apresentado, em consonância com as orientações da escala modificada da dor da OMS, diante da falha da terapia farmacológica com diferentes agentes, indicamos medidas intervencionistas, optando-se pela realização do bloqueio analgésico guiado por ultrassom dos nervos safeno e ciático. A escolha desses dois nervos decorre da região onde o paciente queixava-se de dor mais intensa, ou seja, região medial do pé (inervação do safeno) e região dorso-lateral do pé (inervação do ciático).

O bloqueio do ciático guiado por ultrassonografia pode ser realizado na fossa poplítea e a injeção da solução anestésica pode ser feita na bainha epidural comum que envolve os nervos tibial e fibular comum. O bloqueio do nervo safeno no nível trans-sartorial promove adequada analgesia da porção medial da perna e tornozelo, sem causar fraqueza da musculatura do quadríceps, observado nos bloqueios do safeno em níveis mais altos (perifemoral) e, por isso, foi o nível escolhido.

Apesar de os bloqueios simpáticos proporcionarem redução na dor ao bloquear a contribuição do simpático na SDCR, esse alívio perdura por curto intervalo de tempo na maioria dos pacientes tratados. Isso significa que os pacientes devem continuar sendo submetidos a esses bloqueios frequentemente, como terapia de manutenção.

A terapia intervencionista da dor deve proporcionar um alívio tal da dor que habilite o paciente a realizar fisioterapia para restauração funcional, além de terapia multidisciplinar, não devendo, portanto, atuar como modalidade terapêutica isolada.

E. Risk factors for complex regional pain syndrome in patients with traumatic extremity injury. Ulus Travma Acil Cerrahi Derg. 2010;16:144-8.

7. Xanthos DN, Bennett GJ, Coderre TJ. Norepinephrine-induced nociception and vasoconstrictor hypersensitivity in rats with chronic post-ischemia pain. Pain. 2008;137:640-51.

8. Waldman SD. Comprehensive atlas of ultrasound-guided pain management injection techniques. Philadelphia: Wolter Kluwer; 2014. 1205 p.

9. Waldman SD. Atlas of interventional pain management. 3. ed. Philadelphia: Sauders Elsevier; 2009. 371 p.

10. Szerb J, Persaud D. Long current impulses may be required for nerve stimulation in patients with ischemic pain. Can J Anaesth. 2005;52:963-6. 
11. Wilkes D, Ganceres N, Doulatram G, Solanki D. Alcohol neurolysis of the sciatic and femoral nerves to improve pressure ulcer healing. Pain Pract. 2009;9(2):145-9.
12. Nayak S, Roberts S, Cunliffe M. Management of intractable pain from a chronic dislocated hip in an adolescent. Paediatr Anaesth. 2008;18:357-8.

\section{Como citar:}

Becco CM, Gomes JM. Bloqueio de nervo safeno e nervo ciático para síndrome dolorosa complexa regional tipo II em membro inferior. Rev Med UFC. 2019 abr-jun;59(2):58-61. 